\title{
从工业催化角度看分子篮催化剂未来发展的若干思考
}

\author{
刘志成 ${ }^{1}$, 王仰东 ${ }^{1}$, 谢在库 ${ }^{2,}$, \\ ${ }^{1}$ 中国石油化工股份有限公司上海石油化工研究院, 上海 201208 \\ 2 中国石油化工股份有限公司, 北京 100027
}

摘要: 从工业催化的角度思考和探讨了分子篮催化剂合成、催化及应用方面存在的一些问题与挑战, 并从沸石分子篮的高效催 化、新结构分子篮合成与催化应用、沸石分子篮的经济合成、分子篮在绿色环保领域的新应用等几个方面, 综述了国内外相关 的最新研究进展, 探讨了分子篮催化剂未来的发展方向. 旨在引发人们对分子篮催化未来向经济、可控、高效催化、绿色环保 和新应用等方面发展的思考与探索.

关键词：沸石分子篮; 工业催化剂; 石油化工; 未来发展; 高效催化; 经济合成; 绿色环保

中图分类号: O643 文献标识码: A

收稿日期: 2011-07-29. 接受日期: 2011-09-21.

*通讯联系人. 电话: (021)68462947; 传真: (021)68462283; 电子信箱: xzk@sinopec.com, xiezk2009@gmail.com

基金来源: 国家重点基础研究发展计划 (973 计划, 2009CB623504, 2009CB623506).

本文的英文电子版(国际版)由Elsevier出版社在ScienceDirect上出版(http://www.sciencedirect.com/science/journal/18722067).

\section{Thoughts on the Future Development of Zeolitic Catalysts from an Industrial Point of View}

\author{
LIU Zhicheng ${ }^{1}$, WANG Yangdong ${ }^{1}$, XIE Zaiku ${ }^{2, *}$ \\ ${ }^{1}$ Shanghai Research Institute of Petrochemical Technology, SINOPEC, Shanghai 201208, China \\ ${ }^{2}$ SINOPEC, Beijing 100027, China
}

\begin{abstract}
Problems and challenges in the production and catalysis of zeolitic catalysts were addressed from an industrial point of view. The recent progress and developments in zeolitic catalysts were reviewed. The highly efficient catalysis of zeolitic catalysts, the synthesis and application of novel zeolitic catalysts, the economic production of zeolites, the application of zeolites to environmental protection and green chemistry, etc., were covered. It is intended to stimulate thought and focus on the future development of zeolitic catalysts with regard to economic synthesis, catalyst design, high efficiency catalysis, green processes, new applications, etc.

Key words: zeolite; industrial catalyst; petrochemical industry; development; high efficiency catalysis; economic synthesis; green process
\end{abstract}

Received 29 July 2011. Accepted 21 September 2011.

*Corresponding author.Tel: +86-21-68462947; Fax:+86-21-68462283; E-mail: xzk@sinopec.com,xiezk2009@gmail.com

This work was supported by the National Basic Research Program of China (973 Program, 2009CB623504, 2009CB623506).

English edition available online at Elsevier ScienceDirect (http://www.sciencedirect.com/science/journal/18722067).

化学工业中 $80 \%$ 以上的过程涉及催化技术, 尤 其对于炼油与石化工业, 催化剂更是不可或缺, 而沸 石分子篮是其中重要一员. 沸石分子篮的工业催化 应用始于上世纪 60 年代, Mobil 公司首先发现并采 用八面沸石替代无定形硅铝催化剂, 应用于炼油中 催化裂化 (FCC) 过程, 大大提高了汽油产量以及原
油利用率 ${ }^{[1]}$. 目前, 仅作为 FCC 催化剂一项, 沸石分 子篮催化剂的销售额就占全球催化剂的 $18.5 \%{ }^{[2]}$. 沸石分子篮具有确定的孔体系, 大的晶内比表面积 和与硫酸或氯化铝相当的酸性, 同时具有分子篮分 或择形作用以及可改性或易掺杂等优点, 它们对许 多工业催化反应有高效促进作用, 并已广泛应用于 
催化裂化、加氢裂化、聚合、重整、加氢脱蜡、歧 化、异构化、烷基化、烷基转移、烯烃制汽油、烯 烃裂解、苯与乙烯或丙烯烷基化、低碳烷烃和烯烃 芳构化等炼油与石油化工过程, 以及选择氧化、甲醇 制烯烃、甲醇制汽油等精细化工或煤化工工艺过程 (见表 1). 沸石分子篎的高效催化已给石油化工工 业带来了巨大的经济效益.

然而, 随着世界经济对石化产品需求的不断增 长, 石油资源短缺的矛盾日益突出; 同时, 基本原料 的组成与来源也在不断发生变化, 包括原油中重质 成分越来越高, 煤、天然气或生物乙醇等作为石油替 代资源急需开发与利用等; 此外, 国家与社会对节 能降耗和环保的要求越来越高等等. 这些需求都要
求炼油与石化技术不断革新和发展, 因此, 目前与未 来一段时间将迫切需要发展重质油炼制技术、利用 煤化工等新资源生产石化产品的新技术、低值副产 高效转化技术、特色高附加值石油化工产品生产技 术、低能耗或低物耗技术以及 $\mathrm{CO}_{2}$ 化工利用等技术, 而这些新技术的开发均离不开催化剂的创新与发 展, 其中也包括分子篮催化剂. 从工业催化角度思 考, 围绕资源高效与综合利用、节能降耗、原子经济 和环境友好等目标, 如何进一步提高分子篮催化剂 性能与效率、分子篮催化剂设计与制备是否能达到 可控、分子篮的经济生产工艺、分子篮的催化新应 用等诸多问题是分子篮催化剂研究面临的主要挑 战, 具体来说, 可列举如下:

表 1 沸石分子篮在工业催化中的应用 ${ }^{[3]}$

Table 1 Application of zeolitic catalysts in the petrochemical industry ${ }^{[3]}$

\begin{tabular}{|c|c|c|c|}
\hline Catalytic reaction & Industrial process & Target description & Zeolitic catalyst \\
\hline Catalytic cracking & FCC & $\begin{array}{l}\text { to convert high boiling, high molecular mass } \\
\text { hydrocarbon fractions of petroleum crude oils } \\
\text { to more valuable gasoline, olefinic gases, and } \\
\text { other products }\end{array}$ & REY, USY, ZSM-5 \\
\hline Hydrocracking & MPHC & to produce gasoline with higher quality & NiMo or NiW/USY \\
\hline Gas oil hydrotreating & MIDW & $\begin{array}{l}\text { maximizing production of premium distillate } \\
\text { by catalytic dewaxing }\end{array}$ & $\mathrm{Ni}, \mathrm{W}$ or $\mathrm{Pt} / \mathrm{Y}$ \\
\hline Lube hydrotreating & MDDW, MLDW, MSDW & catalytic dewaxing of gas oil & ZSM-5 \\
\hline Alkane selective cracking & selectoforming & $\begin{array}{l}\text { to produce high octane gasolines from } \\
\text { naphthas }\end{array}$ & erionite \\
\hline $\begin{array}{l}\text { Alkane cracking and alkylation } \\
\text { of aromatics }\end{array}$ & M-reforming & to improve octane and production of gasolines & ZSM-5 \\
\hline n-Alkane hydroisomerization & TIP, CKS & $\begin{array}{l}\text { to improve octane of gasolines by converting } \\
\text { pentane and hexane to isobutane }\end{array}$ & $\mathrm{Pt} / \mathrm{MOR}, \mathrm{Pt} / \mathrm{FRR}$ \\
\hline Olefin oligomerization & MOGD & to convert light olefins to gasoline $\&$ distillate & ZSM-5 \\
\hline Methanol dehydration to gasoline & MTG & to produce gasoline from methanol & ZSM-5 \\
\hline Methanol dehydration to olefins & MTO/MTP, S-MTO/MTP, DMTO & to produce light olefins from methanol & SAPO-34, ZSM-5 \\
\hline $\begin{array}{l}\text { Catalytic cracking of } \mathrm{C}_{4}-\mathrm{C}_{8} \\
\text { olefins }\end{array}$ & $\begin{array}{l}\text { FCFCC, INDMAX, DCC, Superflex, } \\
\text { MOI, PCC, OCP, PROPYLUR, OCC }\end{array}$ & to convert $\mathrm{C}_{4}-\mathrm{C}_{8}$ olefins to light olefins & Y, ZSM-5 \\
\hline Xylene isomerization & $\begin{array}{l}\text { MLPI, MVPI, MHTI, XyMax, } \\
\text { isomar octafining }\end{array}$ & $\begin{array}{l}\text { to convert } m-, o \text {-isomers of xylene or } \\
\text { ethylbenzene to } p \text {-xylene }\end{array}$ & $\mathrm{ZSM}-5, \mathrm{Pt} / \mathrm{Al}_{2} \mathrm{O}_{3} / \mathrm{MOR}$ \\
\hline Toluene disproportionation & Tatoray, MTDP, S-TDT & to produce benzene and xylene from toluene & ZSM-5, MOR \\
\hline $\begin{array}{l}\text { Shape-selective disproportionation } \\
\text { of toluene }\end{array}$ & MSTDP, PxMax , SD & to produce $p$-xylene from toluene & ZSM-5 \\
\hline Heavy aromatics transalkylation & TransPlus, Tatoray, HAP & to produce xylene from $\mathrm{C}_{9+}$ & ZSM-5, MOR, $\beta$ \\
\hline Alkylation of benzene and ethylene & MEB, EBMax, EB One & ethylbenzene production & $\mathrm{ZSM}-5, \mathrm{Y}, \beta, \mathrm{MCM}-22$ \\
\hline $\begin{array}{l}\text { Alkylation of benzene and } \\
\text { propylene }\end{array}$ & $\begin{array}{l}\text { Mobil-Badger, Q-Max, CDcumene, } \\
\text { 3DDM, MP }\end{array}$ & cumene production & $\begin{array}{l}\text { MCM-22, MgSAPO-31, } \\
\text { USY, MOR, B/ } \beta, \\
\text { MCM-56 }\end{array}$ \\
\hline Aromatization & $\begin{array}{l}\text { M2-refroming, cyclar, Z-reforming, } \\
\text { aroforming, pyroform }\end{array}$ & $\begin{array}{l}\text { to produce aromatics from light alkanes and } \\
\text { olefins }\end{array}$ & ZSM-5, Ga/MFI, Pt/KL \\
\hline Selective catalytic oxidation & $\begin{array}{l}\text { epoxidation of propylene } \\
\text { propylene hydration and oxidation } \\
\text { benzene hydration and oxidation }\end{array}$ & $\begin{array}{l}\text { to produce propylene epoxide from propylene } \\
\text { to produce isopropanol from propylene } \\
\text { to produce phenol from benzene }\end{array}$ & TS-1, TS-2, Ti-MWW \\
\hline
\end{tabular}


(1) 实际的工业催化反应千差万别, 另外还面临 市场需求及原料差异等变化, 如何根据催化反应的 特点和要求设计分子篎材料并做到“量体裁衣”是面 临的挑战之一. 其中需要考虑的是孔道结构与大 小、晶粒形貌 (包括大小和形状)、材料组成、酸强 度及其分布等能否做到可控甚至精细可控等问题.

(2) 针对现有工业催化反应, 能否不断提高原有 分子篮的催化效率和性能是面临的挑战之二. 需要 考虑什么样的分子篎孔道结构对提高催化反应性能 有利, 如何提高孔道的有效利用率或催化效率, 孔道 是否可以更加丰富, 分子篮骨架杂原子对催化性能 有何影响, 以及分子篮的表面化学与表面修饰科学 应怎样发展等问题.

(3) 寻找与合成具有更高催化性能的分子篮材 料, 革新原有工艺是分子篮研究面临的挑战之三. 这一点对创新能力的要求很高, 难度也很大, 其中包 括合成水热稳定性高的大孔分子篮、手性分子篮及 特殊结构分子篮等. 目前, 分子篮拓扑结构已达 190 余种, 每种分子篮都各有什么特点、哪种分子篮具有 工业应用前景、采用什么样的分子篮会带来更高的 催化性能等问题都需要思考和研究.

(4) 目前,一些有工业应用前景的沸石分子篮在 合成中使用价格较高的有机胺作结构导向剂, 使得 合成成本较高, 影响其经济性, 如何优化这些沸石分 子篮的合成方法、降低合成成本是值得关注和面临 的挑战之四.

(5) 挑战之五是寻找沸石分子篮催化剂的新用 途, 如在精细化工、环保等方面.

下面就以上这些问题与挑战, 简述国内外相关 的研究进展, 并对其中的科学问题进行一些探讨.

\section{1 沸石分子篮的高效催化}

对于工业催化所用的沸石分子篮而言, 高性能 是基本的要求和目标. 催化材料活性中心的种类与 数量, 以及微孔扩散性能是影响其催化活性的内在 本质因素. 催化选择性则与微孔孔道的择形性、副 反应的发生、各反应分子的扩散快慢有密切关系. 寿命一直是衡量催化材料性能优劣的重要指标, 如 何尽可能地延长催化剂寿命是催化过程的永恒话 题. 在催化剂活性达到要求的前提下, 失活催化剂 如果容易再生, 结构可恢复, 即可以反复再生, 然后
配以合适的反应工艺, 将可以达到延长催化剂寿命 的目的. 所以高性能不仅对沸石分子篎材料提出了 更高的要求, 而且需要催化材料与反应工艺、反应工 程系统进行多尺度的结合与配合, 最终使催化剂在 工业化应用中实现高性能.

以下通过晶粒大小与形状控制、分子篮孔道的 高效利用、分子篎表面修饰功能化等几个方面探讨 提高沸石分子篮催化性能的一些方法或途径.

\section{1 晶粒大小与形状的控制}

大多数沸石分子篮的孔道尺寸小于 $1 \mathrm{~nm}$, 小分 子有机物在分子篮孔道中反应时, 扩散会受到一定 的限制, 从而影响其孔道利用率及催化性能. 减小 晶粒尺寸和改变晶粒形状是提高分子扩散性能和孔 道利用率的手段. 小晶粒或纳米分子篎比大晶粒分 子篮孔道扩散路径短, 其孔道利用率将大大提高, 催 化活性也将有所提高. 因此, 自上世纪九十年代开 始, 纳米沸石的合成与性能研究成为沸石研究领域 的热点之一 ${ }^{[4]}$, 人们利用各种方法先后成功合成出 单分散的 LTA 型 ${ }^{[5]} 、$ LTL 型 ${ }^{[6]} 、$ MFI 型 ${ }^{[7]}$ 和 BEA 型 ${ }^{[8]}$ 等纳米级沸石, 并发现它们在反应活性、产物选择性 和催化剂使用寿命等性能方面较常规沸石更好 ${ }^{[9]}$.

SAPO-34 是甲醇制烯烃 (MTO) 反应的工业分 子篮催化剂。最近, Yang 等 ${ }^{[10]}$ 研究了不同形貌 SAPO-34 分子篎对烯烃收率和催化剂寿命的影响, 实验发现, 分子篮晶粒形貌对催化性能的影响很大, 其中薄片状、小晶粒的分子篮比大晶粒的烯烃收率 更高, 失活速率较慢.

最近, Choi 等 ${ }^{[11]}$ 用双季铵阳离子表面活性剂成 功地合成出了所谓“多层”和“单层”MFI 纳米沸石片, 该材料在甲醇制汽油 (MTG) 反应中积炭失活速率 大大降低.

然而, 小晶粒或纳米沸石在合成和使用过程中 依然面临很多问题, 例如它们在合成中极易团聚, 需 要严格控制合成体系的组成和反应条件. 而在干 燥、高温焙烧等后处理过程中也常常发生团聚, 这样 通常会降低纳米沸石的使用效率. 纳米沸石的合成 过程中常常需要大量价格昂贵的结构导向剂, 造成 合成效率很低, 成本也相应上升. 另外, 在纳米沸石 的使用过程中, 尤其是在液相反应体系中, 催化剂与 产物不易分离, 不利于催化剂的循环使用. 以上难 题都是小晶粒或纳米沸石进一步应用需要解决的. 


\section{2 多级孔复合}

多级孔复合, 尤其是介孔和微孔的复合是近年 来多孔催化材料研究的热点. 目前报道的大部分介 孔材料都存在着材料热稳定性较差, 缺少一定强度 的表面酸性中心及酸中心易流失等缺点, 其主要原 因是尽管上述材料具有有序的介孔孔道, 但其骨架 为无定形结构. 沸石分子篮虽然具有良好的结构稳 定性和较强的酸性中心, 但存在分子扩散的限制, 从 而影响其催化反应的活性和选择性. 而微孔与介孔 或大孔的多级孔复合材料有望结合二者优点并在实 际应用中发挥优势. 近年来, 多级孔复合分子篮已 成为研究热点. 其合成方法主要是在沸石的合成体 系中加入如纳米碳黑 ${ }^{[12]}$ 、纳米碳管 ${ }^{[13]}$ 、淀粉 ${ }^{[14]}$ 、纳 米碳酸钙 ${ }^{[15]}$ 、高分子 ( $\mathrm{PDDA}^{[16]}, \mathrm{PVB}^{[17]}$ 等) 以及含 季铵阳离子有机硅表面活性剂 ${ }^{[18]}$ 等, 使它们与小分 子有机胺模板剂在沸石晶体生长过程中共同作用包 裹于沸石晶体内, 最后焙烧除去这些模板剂即实现 沸石晶体内介孔的生成. 此外, 采用介孔或大孔无 定形墙体转晶的方法也能合成出一些孔壁晶化的介 孔分子篮 ${ }^{[19]}$.

多级孔复合的沸石分子篮在超临界甲苯歧

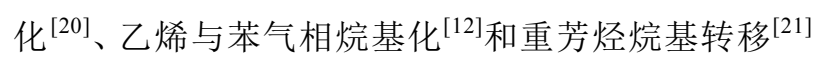
等反应中表现出良好的催化性能. 最近, Yang 等 ${ }^{[22]}$ 采用气相晶化法使介孔-大孔硅铝分子篮转晶合成 得到多级孔 Beta 分子篮, 实验发现, 它在苯与二异 丙苯的液相烷基转移反应中有较好的催化性能. 因 此, 多级孔沸石分子篮有望用于一些较大分子的催 化反应及液相催化反应中.

\section{3 共结晶分子篮}

在合成分子篮时, 同一个反应体系中也常会生 成多种晶体, 这就是分子篮合成中的混晶、共生或共 晶现象, 如常见的 X/A, MFI/MEL, FAU/EMT 和 $\mathrm{STF} / \mathrm{SFF}$ 等共生分子篮. 一些共结晶分子䇻的催化 性能也有较大幅度的提高, 例如 ZSM-5/ZSM-11 (MFI/MEL) 共结晶分子篮应用于 MTG 反应时, 可 大范围调节汽油组分 ${ }^{[23]}$. 共结晶分子篮的催化本质 实际上是孔道与酸性的精细调变, 它是实现提高催 化剂性能的一种手段.

最近, Xie 等 ${ }^{[24]}$ 考察了 MCM-49 转晶法和双有 机胺结构导向剂体系下 MCM-49/ZSM-35 共结晶分 子篮的合成、表征与催化性能. 实验发现, 该共结晶
分子篮结构上不同于机械混合分子篮, 催化裂化汽 油改质反应结果显示, MCM-49/ZSM-35 共结晶分 子篮不是两者的简单迭加, 而是在催化反应中表现 出更强的协同效应, 显示出高于纯 MCM-49 分子篮 的芳构化性能 ${ }^{[25]}$. 此外, 在 1-丁烯的芳构化反应中, MCM-49/ZSM-35 共结晶分子篮中的 MCM-49 与 ZSM-35 同样存在显著的协同效应, 即使其中含少 量的 MCM-49 也可表现出较高的芳构化性能 ${ }^{[26]}$.

祁晓岗等 ${ }^{[27]}$ 也考察了 $\beta$ 沸石 - 丝光沸石 (BEA/MOR) 共结晶分子篎, 实验发现, 共结晶分子 篮与两种纯分子篮机械混合物的结构及酸性质明显 不同.

目前, 对共结晶分子篎的研究还不太多, 在共生 结构、性质及催化性能的特异性研究方面尚有许多 问题亟待研究.

\section{4 分子篮表面修饰与其水热稳定性提高}

热稳定性和水热稳定性是分子篮催化剂需要考 察的重要性质之一, 许多工业催化反应对催化剂的 热稳定性, 尤其是水热稳定性要求很高, 它们往往是 决定催化剂寿命和反应工艺选择的关键.

以碳四烯烃催化裂解反应为例, 由于该反应在 水蒸气条件下进行, 提高催化剂水热稳定性是碳四 烯烃催化剂开发的关键. 结果表明, 通过对多孔材 料催化活性中心进行磷氧化合物组装修饰、引入骨 架杂原子等, 可以提高催化材料在水蒸气条件下活 性中心的稳定性 ${ }^{[28]}$. Zhao 等 ${ }^{[29]}$ 将 ZSM-5 分子篮浸 以磷酸后在一定温度下焙烧引入适量的磷氧化合物 改性. 实验结果表明, 催化剂的水热稳定性得到提 高, 并且有效减缓积炭失活速率, 而且孔道中氧化物 物种与骨架 $\mathrm{Si}-\mathrm{O}-\mathrm{Al}$ 的协同作用使多孔催化材料酸 中心的强度和分布得到优化, 减少了氢转移等副反 应的发生, 提高了丙烯选择性. 为了解释分子篮经 磷氧化合物改性后水热稳定性提高的原因, Xue 等 ${ }^{[30]}$ 研究了水蒸气处理前后不同磷含量的 P/HZSM-5 的 $\mathrm{D}_{2} / \mathrm{OH}$ 交换情况, 并通过分子模拟计 算研究了磷与 HZSM-5 相互作用的模型. 结果发现, 对于水蒸气处理前的 P/HZSM-5, 其酸性可能来自 较弱的 $\mathrm{P}-\mathrm{OH}$ 酸位, 且随磷含量的增加, B 酸位减少 的趋势逐渐减缓; 高温水蒸气处理过程伴随着脱 铝、磷的少量挥发、磷与铝物种的结合以及高温下 $\mathrm{P}$ 物种之间的缩合, 即样品在高温水蒸气处理过程 
中生成了一种不同于分子篮中的桥式羟基, 也不同 于 $\mathrm{P}-\mathrm{OH}$ 的新酸位, 这些过程导致 P/HZSM-5 酸性 质的变化, 表面羟基密度降低, 水热稳定性提高.

此外, 对于 MTO 反应, 产物中有大量的水生成, 因此分子篎催化剂是否耐高温水热和反复高温再 生, 是选择该催化剂的关键. 正因为 SAPO-34 分子 笁热稳定性和水热稳定性良好, 它已成为 MTO 催 化剂的首选材料.

另外, 对轻油裂解制低碳烯烃反应来说, 反应温 度通常在 $600{ }^{\circ} \mathrm{C}$ 以上, 对催化剂热稳定性和水热稳 定性要求很高, 需要寻找活性更高、水热稳定性更好 的催化材料.

其它还有许多炼油与石油化工催化反应, 包括 炼油中的催化裂化反应都需要热稳定和水热稳定性 高的催化材料或载体, 寻找具有高温水热稳定性的 多孔材料及提高水热稳定性表面修饰方法是需要关
注和研究的重点.

\section{2 新结构分子節的合成与催化应用}

\section{1 大孔与超大孔分子笠}

尽管沸石类催化剂在石油化工和精细化工中的 应用已经取得了很大的进展, 但由于许多分子太大 (如重质原油组分、精细化学品和中间体分子等) 而 无法进入沸石孔道内, 不能达到预期的催化效果甚 至不能反应. 因此, 制备具有大孔 (12 元环) 或超大 孔 (大于 12 元环) 多维结构的沸石及沸石类分子篮 材料已成为近十年来人们努力追求的一个重要目标 (见表 2).

磷酸盐分子篮 VPI-5 是第一例孔径超过 $1 \mathrm{~nm}$ 的分子篮, 它具有单一的 $1.2 \mathrm{~nm}$ 的孔径分布和 $30 \%$ 的空腔比例. 它的出现开辟了超大孔分子篮的合成 方向, 随后一些超大孔的磷酸盐、硅酸盐、硅锗酸盐、

表 2 大孔与超大孔分子耖

Table 2 Microporous materials with large or super large pore

\begin{tabular}{|c|c|c|c|c|c|}
\hline Material & Year reported & Main framework composition & Ring size (TO) & Pore size $(\mathrm{nm})$ & Reference \\
\hline VPI-5 & 1988 & $\mathrm{AlPO}_{4}$ & 18 & 1.2 & {$[31]$} \\
\hline $\mathrm{AlPO}_{4}-8$ & 1990 & $\mathrm{GaPO}_{4}$ & 14 & $<1.0$ & {$[32,33]$} \\
\hline Cloverite & 1991 & $\mathrm{GaPO}_{4}$ & 20 & $<1.0$ & [34] \\
\hline JDF-20 & 1992 & $\mathrm{AlPO}_{4}$ & 20 & collapse upon activation & {$[35]$} \\
\hline ULM-5 & 1994 & $\mathrm{GaPO}_{4}$ & 16 & not reported & {$[36]$} \\
\hline AIMepO- $\beta$ & 1995 & $\mathrm{Al}_{2}\left(\mathrm{CH}_{3} \mathrm{PO}_{3}\right)_{3}$ & 18 & 0.6 & {$[37]$} \\
\hline Not named & 1996 & $\mathrm{~V}_{5} \mathrm{O}_{9}\left(\mathrm{PO}_{4}\right)_{2}$ & 16 & not reported & {$[38]$} \\
\hline ULM-16 & 1996 & $\mathrm{GaPO}_{4}$ & 16 & not reported & [39] \\
\hline UTD-1 & 1996 & $\mathrm{SiO}_{2}$ & 14 & 1.0 & {$[40]$} \\
\hline ULM-15 & 1997 & $\mathrm{FePO}_{4}$ & 16 & not reported & [41] \\
\hline CIT-5 & 1997 & $\mathrm{SiO}_{2}$ & 14 & 1.0 & {$[42]$} \\
\hline ASU-31 & 1999 & $\mathrm{InS}_{4}$ & 24 & 2.56 & {$[43]$} \\
\hline ASU-32 & 1999 & $\mathrm{InS}_{4}$ & 24 & 1.47 & [43] \\
\hline VSB-1 & 1999 & $\mathrm{NiPO}_{4}$ & 24 & 0.9 & {$[44]$} \\
\hline ND-1 & 1999 & $\mathrm{ZnPO}_{4}$ & 24 & 1.48 & {$[45]$} \\
\hline VSB-5 & 2001 & $\mathrm{NiPO}_{4}$ & 24 & 1.0 & {$[46]$} \\
\hline FDU-4 & 2001 & $\mathrm{GeO}_{2}$ & 24 & 1.26 & {$[47]$} \\
\hline NTHU-1 & 2001 & $\mathrm{GaPO}_{4}$ & 24 & 1.1 & {$[48]$} \\
\hline OSB-1 & 2001 & $\mathrm{Si}_{0.66} \mathrm{Be}_{0.33} \mathrm{O}_{2}$ & 14 & 0.73 & [49] \\
\hline ITQ-21 & 2002 & $\mathrm{Si}(\mathrm{Ge}) \mathrm{O}_{2}$ & 12 & 1.18 & {$[50]$} \\
\hline ITQ-24 & 2003 & $\mathrm{Si}(\mathrm{Ge}) \mathrm{O}_{2}$ & 12 & 0.68 & {$[51]$} \\
\hline SSZ-53 & 2003 & $\mathrm{~B}_{1.6} \mathrm{Si}_{62.4} \mathrm{O}_{128}$ & 14 & 0.87 & {$[52]$} \\
\hline SSZ-59 & 2003 & $\mathrm{~B}_{0.35} \mathrm{Si}_{15.65} \mathrm{O}_{32}$ & 14 & 0.85 & {$[52]$} \\
\hline ECR-34 & 2003 & $\mathrm{Si}_{0.75} \mathrm{Ga}_{0.24} \mathrm{Al}_{0.01} \mathrm{O}_{2}$ & 18 & 1.01 & {$[53]$} \\
\hline IM-12 & 2004 & $\mathrm{Si}(\mathrm{Ge}) \mathrm{O}_{2}$ & 14 & 0.95 & {$[54]$} \\
\hline ITQ-33 & 2006 & $\mathrm{Si}(\mathrm{Ge}) \mathrm{O}_{2}$ & 18 & 1.22 & {$[55]$} \\
\hline ITQ-37 & 2009 & $\mathrm{Si}(\mathrm{Ge}) \mathrm{O}_{2}$ & 30 & 1.93 & {$[56]$} \\
\hline ITQ-40 & 2010 & $\mathrm{Si}(\mathrm{Ge}) \mathrm{O}_{2}$ & 16 & not reported & {$[57]$} \\
\hline ITQ-44 & 2010 & $\mathrm{Si}(\mathrm{Ge}) \mathrm{O}_{2}$ & 18 & 1.25 & {$[58]$} \\
\hline ITQ-43 & 2011 & $\mathrm{Si}(\mathrm{Ge}) \mathrm{O}_{2}$ & 23 & 2.19 & {$[59]$} \\
\hline
\end{tabular}


硫化物分子篮被合成出来 (如表 2 所示), 其中, 超大 孔磷酸盐分子篎材料的热稳定性较差, 如 JDF-20 和 ND-1, 它们在除去客体分子后孔道坍塌或转晶成致 密相, 而超大孔的硅酸盐沸石分子篮热稳定性则较 好, 如 UTD-1 和 CIT-5 就与其它小孔沸石分子篮热 稳定性相当 ${ }^{[42]}$. 因此, 从热稳定性来看, 大孔、超大 孔硅酸盐分子篮比超大孔磷酸盐分子篮应该有更为 广阔的工业应用前景.

值得一提的是, 近年来 Corma 研究组将 $\mathrm{Ge}$ 引 入到分子篮结构中得到一系列的含 $\mathrm{Ge}$ 分子篮, 其 中包括一些大孔、超大孔分子篮, 如 ITQ-21 $1^{[50]}$, ITQ-24 $4^{[51]}$, ITQ-33 ${ }^{[55]}$, ITQ-43 ${ }^{[59]}$ 和 ITQ-44 ${ }^{[58]}$ 等. 其 中, ITQ-21 分子篮拥有三维互联小孔结构和含有较 大的笼状结构, 每个笼状结构都可经由 6 个大窗口 进入, 它作为石油裂化催化剂不仅可增加轻油收率, 而且其石油产品辛烷值较高 ${ }^{[50]}$. ITQ-33 具有 18 元 环孔口和双十元环交叉孔道体系, 它对于烷基化和 烷基芳烃的烷基转移活性非常高, 对于含有单环或 双环稠环芳环的大取代基烷基芳烃的脱烷基的活性 也非常高. 在 VGO 催化裂化过程中, 它可产生更多 的柴油和更少的汽油, 同时保持丙烯和丁烯产率 ${ }^{[55]}$. 所以, 如果稳定性和经济性得到进一步提高, 诸如 ITQ-21 和 ITQ-33 等大孔或超大孔分子篮在炼油和 石油化工中将有很大的工业前景.

\section{2 手性分子篮}

目前高选择性反应, 特别是不对称催化合成反 应已经成为研究的焦点之一, 而且在制药、发展农作 物、农业化学品、食物添加剂等合成工业上都有重 要的应用前景. 以沸石分子篮为催化材料进行不对 称合成反应时, 往往需要以分子篮为主体, 固载具有 不对称结构的配合物和金属有机物等, 以制成新的 手性催化剂. 然而, 这样制成的手性催化剂只能通 过桥键或辅助试剂引入手性, 所以催化剂的稳定性 及催化寿命一直阻碍着其进一步的工业开发应用. 如果带有手性结构的分子篮材料能够被合成出来, 其稳定性的问题就可能迎刃而解. Davis 等 ${ }^{[60]}$ 通过 加入具有手性的结构导向剂合成出手性 $\beta$ 沸石, 但光 活性实验结果证实只得到 $5 \%$ 的对映选择性. Stucky 研究小组合成出了具有手性结构的 $\mathrm{NaZnPO}_{4}{ }^{[61]}$ 和 $\mathrm{NaCoPO}_{4}{ }^{[62]}$ 及具有三维螺旋形孔的 锗镓分子篮 UCSB-7 $7^{[63]}$. 最近, Zou 研究小组 ${ }^{[64]}$ 又合
成出了两种手性锗硅分子篮 SU-15 和 SU-32. 尽管 这些材料目前还未被应用, 但为分子篮手性催化的 实现建立了良好的研究基础.

\section{3 特殊结构分子篮}

MWW 型沸石分子篎是一类新型的具有双孔道 系统的层状结构高硅沸石, 家族成员包括 MCM-22, MCM-49, MCM-36, MCM-56, PSH-3, SSZ-25, ERB-1, ITQ-1, ITQ-2, EMM-10, EMM-12 和 EMM-13 等 ${ }^{[65]}$, 它的层内包含二维、正弦及交叉的十 元环粗圆形孔道系统, 层间为十二元环超笼, 与传统 沸石分子篮不同, 其表面存在较大的孔穴. 因此, 它 在烯烃异构化、芳烃烷基化为乙苯或异丙苯、催化 裂化及加氢裂化、甲苯歧化以及芳构化等许多反应 中均表现出优异的催化性能 ${ }^{[66]}$. 近二十年来, MWW 分子篮的研究、开发与应用一直是分子篮领域关注 的热点. Mobil 公司在 MWW 型沸石催化剂基础上 推出了 Mobil-Badger 液相烷基化制异丙苯新技术 ${ }^{[67]}$, 催化过程不仅反应温度低, 而且催化剂寿命长, 选择性高, 异丙苯选择性达到 $99.99 \%$ 以上. 该技术 在全世界得到了迅速推广, 取代了基于 ZSM-5 和 $\beta$ 分子篮的异丙苯制造技术. 另外, MWW 型沸石分 子篮催化剂也已用于乙苯的工业化生产 ${ }^{[68]}$.

EU-1 分子篎 (EUO) 含有十元环直通孔道及与 之垂直联通的十二元环侧袋 (Side Pocket) 结构, 这 种特殊结构使它在二甲苯异构化反应中表现出更高 的活性和选择性 ${ }^{[69]}$, 因而具有应用开发前景.

具有非交叉、一维孔道的中孔沸石, 如 TON, MTT, EUO 和 AEL 等, 作为双功能烷烃加氢异构化 催化剂, 比传统的 ZSM-5, ZSM-11 或 $\beta$ 沸石具有更 高的产率和产物选择性, 因而具有一定的应用开发 前景 ${ }^{[70,71]}$.

\section{3 沸石分子篮的经济合成}

有些沸石分子篮在无机阳离子体系中直接合 成, 如 $A, P$, 丝光沸石, $X, Y$ 和 $L$ 等沸石, 而有些往 往需要在有机胺 (铵) 体系下合成, 如 ZSM-5, ZSM-11, $\beta$ 沸石, MCM-2, SAPO-34 和 TS-1 等. 相 比较而言, 有机阳离子作为模板剂合成出的沸石产 品在结晶度、晶粒大小和硅铝比等产品质量上更加 容易控制, 但原料成本相对较高. 此外, 许多新类型 的分子篮在发现之初往往由比较昂贵的有机胺 (铵) 
来导向合成, 成本很高. 因此, 世界各国的科学家也 一直在努力研究用廉价的及环境友好的结构导向剂 来合成分子篮, 其中包括有机胺替代法和无胺合成 法等.

\section{1 有机胺替代法}

按以往分子篮的合成经验, 人们认识到, 在许多 有机胺 (铵) 合成分子篮的体系中, 有机胺用量往往 是过量的, 大大超过产物分子篮结构中包含的有机 胺量, 这意味着只有少量有机胺真正起结构导向作 用参与分子篮的成核与生长, 而大部分有机胺只是 起到调节碱性和孔道填充的作用. 正是利用这点, 近年来 Zones 等 ${ }^{[72]}$ 研究和发展了有机胺替代法, 即 在合成中加入少量作结构导向剂的有机胺外, 同时 加入其它廉价的有机胺作碱性调节和孔道填充剂. 例如, 他们使用便宜的异丁胺部分替代季铵化的金 刚烷胺合成了 SSZ-25 (MWW) 分子篮, 从而大大降 低了合成成本、有机胺处理成本和晶化时间. 类似 地, 他们用 $N, N, N$-三甲基苯铵代替部分的特殊金刚 烷胺合成出 SSZ-13 (CHA), SSZ-33 (CON), SSZ-35

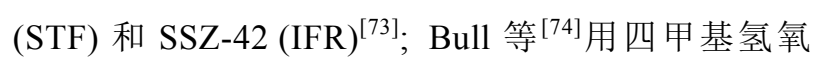
化铵与金刚烷胺组合也合成了 SSZ-13. 值得一提的 是, 双有机胺法还可改变分子篮的结构组成、硅铝比 和晶粒大小等. 例如, 对于 SSZ-47 分子篮来说, 它 是 EUO, NES 和 NON 三种结构的共生, 而其中 NON 结构的存在可大大降低分子篮吸附容量和分 子扩散速率. Lee 等 ${ }^{[75]}$ 用双有机胺法合成了 SSZ-47B, 与采用单有机胺合成的 SSZ-47 相比, SSZ-47B 的 NON 结构含量大大降低, 因此其吸附 容量和分子扩散速率也大大提高. 用双有机胺法合 成的 SSZ-32X (MTT) ${ }^{[76]}$ 比仅用咪唑胺合成的 SSZ-32 分子篮硅铝比更低, 晶粒更小, 其催化脱蜡 的性能也更佳.

另外, 工业上应用的 MCM-22 (MWW) 沸石一 般采用六亚甲基亚胺为结构导向剂来生产. Lewis 等 ${ }^{[77 ~ 80]}$ 用工业上常用的有机胺组合合成了 UZM 系 列沸石分子篮. 其中, 采用二乙基二甲基氢氧化铵 为结构导向剂合成 UZM-8 (MWW) 沸石时 ${ }^{[80]}$, 较金 刚烷胺或六亚甲基亚胺为结构导向剂, 在合成成本 或环境友好性方面有一定优势.

Wang 等 ${ }^{[11]}$ 采用价格低廉且对环境友好的四甲 基胍代替有机胺合成出 AFI 结构的磷铝及其杂原子
分子篮.

\section{2 无胺法合成}

作为工业应用的沸石分子篮, 从生产和成本考 虑, 希望它们用廉价的或纯粹不用有机结构导向剂 条件下就可以合成.

以往采用加入晶种的方法可使有机结构导向剂 的用量大大较低, 例如对于 EU-1 ${ }^{[82]}, \mathrm{ZSM}-11^{[83]}$, $\mathrm{ZSM}-12^{[84]}, \mathrm{SSZ}-28^{[85]}, \mathrm{SSZ}-32^{[86]}$ 和 SSZ-35 ${ }^{[87]}$ 等分 子篮, $\mathrm{SDA} / \mathrm{SiO}_{2}$ 摩尔比可降低到 $0.02 \sim 0.05$. 但是否 能够完全不用有机结构导向剂, 是一个值得关注和 研究的课题.

早在上世纪 80 年代初, 李赫咺和项寿鹤等 ${ }^{[88]}$ 首次提出在无有机模板条件下合成 ZSM-5 的路线, 并成功应用于工业生产中. 王福生等 ${ }^{[89]}$ 和 Clearfield 等 ${ }^{[90]}$ 也在氨水存在的条件下, 以无定形硅酸铝 为原料成功合成出 ZSM-5. 近几年来, 肖丰收等 ${ }^{[91]}$ 对无胺法进行了新的发展和探索, 他们在无胺的合 成体系中同时加入分子篮晶种或凝胶导向剂, 从而 诱导或促使分子篮在无胺体系中生长. 例如, 他们 使用 $\mathrm{Y}$ 型分子篎凝胶导向剂成功地合成了 ECR-1 沸石 ${ }^{[22]}$; 用 $\mathrm{L}$ 型凝胶导向剂合成出了 $\mathrm{ZSM}-34$ 沸 石 ${ }^{[93]}$; 还用炦烧过的 Beta 沸石晶种在无胺体系下 合成了具有较高结晶度的 Beta 沸石 ${ }^{[94]}$, 尽管产品硅 铝比较低, 但随后 Kamimura 等 ${ }^{[95]}$ 进一步拓展了其 合成硅铝比范围, 并且详细研究了其晶化过程, 发现 Beta 沸石晶种并没有诱导成核而是起到提供表面生 长的作用 ${ }^{[96]}$.

据统计, 到目前为止, 富铝的 FAU, $\mathrm{MOR}^{[97]}$, $\mathrm{AMA}$ 和 $\mathrm{CHA}$ 类型分子篮及富硅的 ZSM-5, TUN-7 ${ }^{[98]}$, ECR-1 $^{[92]}$, ZSM-34 $^{[93]}$, Ferrierite ${ }^{[99]}$, $\mathrm{Beta}^{[94,95]}, \mathrm{RTH}^{[100]}$ 和 $\mathrm{MTW}^{[101]}$ 等分子篮均可在无有 机结构导向剂体系下合成.

无胺法合成沸石分子篮能大大降低原料成本, 在经济和环保方面有潜在的优势, 但也面临一些问 题或挑战: (1) 虽然合成体系加入了晶种, 但如要使 产品达到较高的结晶度 (或产率), 需要的晶化时间 相对比较长, 因为往往晶化时间短则结晶度 (或产 率) 不够高; (2) 与有机胺 (铵) 合成法相比, 无胺法 合成的沸石分子篮硅铝比范围往往较窄, 如何拓宽 硅铝比范围是一个挑战; (3) 由于缺少有机胺 (铵) 结构导向剂的帮助, 往往比较容易生成杂晶, 因此, 
如何控制和避免生成杂晶也是问题之一; (4) 晶粒 大小的控制. 如果能解决这些问题, 无胺法生产将 会实现工业应用. 据报道目前已有一些沸石的无胺 法生产进入商业化阶段 ${ }^{[100]}$.

\section{4 新应用领域拓展}

随着分子篎研究的不断深入和扩展, 人们发现 在一些分子篮气体分离、选择氧化和选择性催化还 原脱 $\mathrm{NO}_{x}(\mathrm{SCR})$ 等绿色环保领域具有新的用途.

ETS-4 是 Kuznicki ${ }^{[102]}$ 于 1989 年发现的钛硅分 子篮, 其骨架是由四面体配位的硅原子和八面体配 位的钛原子构成. 后经研究发现, ETS-4 分子篮的 脱水过程可使孔径发生系统的、均匀的和可控的收 缩, 此现象被称为“分子门效应”(molecular gate effect $)^{[103]}$, Engelhard 公司利用 ETS-4 分子篮的这种 特殊性质已将其应用于天然气管道输送中, 分离去 除天然气中的 $\mathrm{N}_{2}$ 和 $\mathrm{CO}_{2}$ 杂质.

另外, 具有选择氧化催化功能的钛硅分子篮, 如 $\mathrm{TS}-1^{[104]}, \mathrm{TS}-2^{[105]}$ 和 Ti-MWW 等, 已被用于催化 $\mathrm{H}_{2} \mathrm{O}_{2}$ 氧化苯酚制对苯二酚、环己酮氨氧化制环己酮 肜以及丙烯环氧化等过程, 实现了这些反应过程的 绿色化. 近 20 年来, 人们主要围绕新类型钛硅分子 篮的探索 (如 Ti-beta ${ }^{[106 ~ 108]}$, Ti-ZSM-12 $2^{[109]}$, Ti-ZSM-48 ${ }^{[110]}$, Ti-mordenite ${ }^{[111]}$, Ti-SSZ-33 ${ }^{[112]}$, Ti-ITQ-7 ${ }^{[113]}$, Ti-MCM-22 (Ti-MWW) ${ }^{[114 \sim 116]}$ 和 Ti-MCM-56 ${ }^{[117]}$ 等), 如何提高分子篮骨架中四配位 钛的含量 ${ }^{[118]}$, 以及钛硅分子篮的新应用等方面展开 研究. 中石化石油化工科学研究院及华东师范大学 也在空心钛硅分子篎 ${ }^{[119]}$ 及 $\mathrm{Ti}-\mathrm{MWW}{ }^{[120]}$ 等新型铁硅 分子篮合成与工业应用方面开展了许多工作, 我们 相信, 铁硅分子篮等在绿色化工等领域的应用将会 越来越广.

此外, 沸石分子篮作为高效的催化剂在环保等 领域也有潜在的应用开发价值. 例如, 如何高选择 性地还原消除贫燃发动机和柴油机尾气中的 $\mathrm{NO}_{x}$ 是世界瞩目的研究课题. 而含 $\mathrm{Cu}, \mathrm{Fe}$ 和 $\mathrm{V}$ 等过渡金 属离子的分子篮催化剂, 如 $\mathrm{Cu} / \mathrm{SAPO}-34^{[121]}$, $\mathrm{Cu} / \mathrm{Beta}$ 或 $\mathrm{Fe} / \mathrm{Beta}^{[122]}$ 和 $\mathrm{Cu} / \mathrm{ZSM}-5^{[123]}$ 等, 对 $\mathrm{SCR}$ 反应的催化活性高, 选择性好, 近年来已成为分子篮 催化领域研究的热点 ${ }^{[124]}$. 我们也期待分子篮有更 多新的应用.

\section{5 结语与展望}

分子篎催化在工业上的应用已有 50 多年的历 史, 沸石分子篮在工业催化上尤其是在许多炼油与 石化过程中占有相当的比例并发挥着非常大的作 用, 使许多石化过程实现了高效率转化或原子经济 转化. 进入 21 世纪以来, 石油化工的发展面临资源 短缺的巨大挑战, 采用新工艺、新技术、新材料, 追 求石油化工过程的节能降耗已经成为石油化工发展 的必然趋势, 同时开发以煤炭、天然气、生物质原料 制备石油化工产品的新工艺以解决资源短缺问题是 近年来的研究热点. 另外, 清洁生产、环保与温室气 体减排与转化也是当今国家关注的重点. 面对这些 国家、行业和市场的发展需求, 分子篮催化面临着如 何进一步提高催化剂性能与效率、催化剂设计与制 备是否达到可控、如何进一步提高合成经济性、分 子篮的催化新应用等诸多问题和挑战. 国内外分子 篮研究者也正围绕这些问题和难点在努力探索和研 究.

我们相信, 随着时间的推移和人们对分子篮研 究的不断深入和发展, 各种新结构分子篮材料将不 断涌现, 不仅丰富了已有的分子篮多孔材料, 更为实 现催化过程的工业化目标提供了更多的选择, 许多 新类型的性能更高的分子篮将替代许多传统的分子 篮催化材料, 帮助石化工艺过程实现高效率转化或 原子经济转化. 许多新的反应工艺也将随着新催化 材料的开发应运而生. 而对于传统的分子篮来说， 对晶粒大小和形貌控制技术水平大大提高, 纳米分 子篮、片状分子篮、针状分子篮和多级孔分子篮等 将用于原有催化剂的改进和升级. 另外, 我们也相 信将来会有若干手性分子篮得到成功开发, 它将应 用于精细化工、药物合成、农药和分离等领域, 从而 成为石油化工行业新的增长点. 此外, 随着合成方 法、工艺与设备的改进, 以及分子篮合成成本的降 低, 将有更多的新类型分子篮实现商业应用. 除了 石化行业, 我们认为沸石分子篮在诸如选择氧化和 $\mathrm{SCR}-\mathrm{NO}_{x}$ 等绿色化工与环保等领域将有越来越多的 新的应用.

\section{参 考 文 献}

1 Plank C J. In: Davis B H, Hettinger W P Jr eds. Heterogeneous Catalysis, Selected American Histories, ACS Sym- 
posium Series 222. Washington: American Chemical Society, 1983. 253

2 Vermeiren W, Gilson J P. Top Catal, 2009, 52: 1131

3 谢在库. 新结构高性能多孔催化材料. 北京: 中国石化 出版社 (Xie Z K. Porous Catalytic Materials With New Structure And Improved Performance. Beijing: Sinopec Press), 2010. 4

4 王亚军, 唐颐, 王星东. 石油化工 (Wang Y J, Tang Y, Wang X D, Petrochem Technol), 2002, 31: 217

5 Schoeman B J, Sterte J, Otterstedt J E. Zeolites, 1994, 14: 110

6 Tsapatsis M, Lovallo M, Okubo T, Davis M E, Sadakata M. Chem Mater, 1995, 7: 1734

7 Persson A E, Schoeman B J, Sterte J, Otterstedt J E. Zeolites, 1995, 15: 611

8 Camblor M A, Corma A, Mifsud A, Perez-Pariente J, Valencia S. Stud Surf Sci Catal, 1997, 105: 341

9 柳云骐, 刘春英, 刘晨光. 化学进展 (Liu Y Q, Liu Ch Y, Liu Ch G. Progress Chem), 2005, 17: 666

10 Yang H Q, Liu Zh Ch, Gao H X, Xie Z K. J Mater Chem, 2010, 20: 3227

11 Choi M, Na K, Kim J, Sakamoto Y, Terasaki O, Ryoo R. Nature, 2009, 461: 246

12 Christensen C H, Johannsen K, Schmidt I, Christensen C H. J Am Chem Soc, 2003, 125: 13370

13 Schmidt I, Boisen A, Gustavsson E, Stahl K, Pehrson S, Dahl S, Carlsson A, Jacobsen C J H. Chem Mater, 2001, 13: 4416

14 刘志成, 孔德金, 王仰东, 谢在库. 石油学报 (石油加 工)( Liu Zh Ch, Kong D J, Wang Y D, Xie Z K. Acta Petrol Sin (Petrol Process Sect)), 2008, 24(Z1): 124

15 Zhu H B, Liu Zh Ch, Wang Y D, Kong D J, Yuan X H, Xie Z K. Chem Mater, 2008, 20: 1134

16 Xiao F Sh, Wang L F, Yin C Y, Lin K F, Di Y, Li J X, Xu R R, Su D S, Schlögl R, Yokoi T, Tatsumi T. Angew Chem, Int $E d, 2006$, 45: 3090

17 Zhu H B, Liu Zh Ch, Kong D J, Wang Y D, Yuan X H, Xie Z K. J Colloid Inter Sci, 2009, 331: 432

18 Choi M, Cho H S, Srivastava R, Venkatesan C, Choi D H, Ryoo R. Nature Mater, 2006, 5: 718

19 Fan W, Snyder M A, Kumar S, Lee P S, Yoo W C, McCormick A V, Lee Penn R, Stein A, Tsapatsis M. Nature Mater, 2008, 7: 984

20 刘志成, 王仰东, 孔德金, 谢在库. 见: 王静康 主编. 现代化工、治金与材料技术前沿, 中国工程院第七届学 术会议论文集 (上册). 北京: 化学工业出版社 (Liu Zh Ch, Wang Y D, Kong D J, Xie Z K. In: Wang J K ed. Frontiers of Modern Chemical Engineering, Metallurgy, and Material Technologies: Proceedings of the 7th Chinese Academy of Engineering Symposium. Beijing: Chem Ind Press), 2009. 557

21 Zhu H B, Liu Zh Ch, Kong D J, Wang Y D, Xie Z K. J Phys Chem C, 2008, 112: 17257

22 Yang H Q, Liu Zh Ch, Gao H X, Xie Z K. Appl Catal A,
2010, 379: 166

23 Thomas J, Ramdas S, Millward B. New Scientist, 1982, 96: 435

24 Xie S J, Liu Sh L, Liu Y, Li X J, Zhang W P, Xu L Y. Microporous Mesoporous Mater, 2009, 121: 166

25 Niu X L, Song Y Q, Xie S J, Liu Sh L, Wang Q X, Xu L Y. Catal Lett, 2005, 103: 211

26 谢素娟, 刘盛林, 张玲, 徐龙伢. 石油学报 (石油加 工)(Xie S J, Liu Sh L, Zhang L, Xu L Y. Acta Petrol Sin (Petrol Process Sect)), 2009, 25(S1): 37

27 祁晓岗, 李斌, 李士杰, 刘希尧, 林炳雄. 催化学报 (Qi X L, Li B, Li Sh J, Liu X Y, Lin B X. Chin J Catal), 2006, 27: 228

28 Kaeding W W, Chu C, Young L B, Weinstein B, Butter S A. J Catal, 1981, 67: 159

29 Zhao G L, Teng J W, Xie Z K, Jin W Q, Yang W M, Chen Q L, Tang Y. J Catal, 2007, 248: 29

30 Xue N H, Chen X K, Nie L, Guo X F, Ding W P, Chen Y, Gu M, Xie Z K. J Catal, 2007, 248: 20

31 Davis M E, Saldarriaga C, Montes C, Garces J, Crowder C. Nature, 1988, 331: 698

32 Dessau R M, Schlenker J L, Higgins J B. Zeolite, 1990, 10: 522

33 Vogt E T C, Richardson J W Jr. J Solid State Chem, 1990, 87: 469

34 Estermann M, McCusker L B, Baerlocher C, Merrouche A, Kessler H. Nature, 1991, 352: 320

35 Huo Q S, Xu R R, Li S G, Ma Z G, Thomas J M, Jones R H, Chippindale A M. J Chem Soc, Chem Commun, 1992: 875

36 Loiseau T, Ferey G. J Solid State Chem, 1994, 111: 403

37 Maeda K, Akimoto J, Kiyozumi Y, Mizukami F. Angew Chem, Int Ed, 1995, 34: 1199

38 Khan M I, Meyer L M, Haushalter R C, Schweitzer A L, Zubieta J, Dye J L. Chem Mater, 1996, 8: 43

39 Loiseau T, Ferey G. Mater Res Soc Confer Proc, 1996, 431: 27

40 Freyhardt C C, Tsapatsis M, Lobo R F, Balkus K J Jr, Davis M E. Nature, 1996, 381:295

41 Cavellec M, Greneche J M, Riou D, Ferey G. Microporous Mater, 1997, 8: 103

42 Wagner P, Yoshikawa M, Tsuji K, Davis M E, Lovallo M, Taspatsis M. Chem Commun, 1997: 2179

43 Li H L, Laine A, O’Keeffe M, Yaghi O M. Science, 1999, 283: 1145

44 Guillou N, Gao Q M, Nogues M, Morris R E, Hervieu M, Ferey G, Cheetham A K. C R Acad Sci, Ser IIc, 1999, 2: 387

45 Yang G Y, Sevov S C. J Am Chem Soc, 1999, 121: 8389

46 Guillou N, Gao Q M, Forster P M, Chang J-S, Nogues M, Park S-E, Ferey G, Cheetham A K. Angew Chem, Int Ed, 2001, 40: 2831

47 Zhou Y M, Zhu H G, Chen Z X, Chen M Q, Xu Y, Zhang H Y, Zhao D Y. Angew Chem, Int Ed, 2001, 40: 2166

48 Lin C-H, Wang S-L, Lii K-H. J Am Chem Soc, 2001, 123: 4649

49 Cheetham A K, Fjellvg H, Gier T E, Kongshaug K O, 
Lillerud K P, Stucky G D. Stud Surf Sci Catal, 2001, 135: 158

50 Corma A, Diaz-Cabanas M J, Martinez-Triquero J, Rey F, Rius J. Nature, 2002, 418: 514

51 Castaneda R, Corma A, Fornes V, Rey F, Rius J. J Am Chem Soc, 2003, 125: 7820

52 Burton A, Elomari S, Chen C-Y, Medrud R C, Chan I Y, Bull L M, Kibby C, Harris T V, Zones S I, Vittoratos E S. Chem Eur J, 2003, 9: 5737

53 Strohmaier K G, Vaughan D E W. J Am Chem Soc, 2003, 125: 16035

54 Paillaud J-L, Harbuzaru B, Patarin J, Bats N. Science, 2004, 304: 990

55 Corma A, Díaz-Cabañas M J, Jordá J L, Martínez C, Moliner M. Nature, 2006, 443: 842

56 Sun J L, Bonneau C, Cantin A, Corma A, Diaz-Cabanas M J, Moliner M, Zhang D L, Li M R, Zou X D. Nature, 2009, 458: 1154

57 Simancas R, Dari D, Velamazán N, Navarro M T, Cantín A, Jordá J L, Sastre G, Corma A, Rey F. Science, 2010, 330: 1219

58 Jiang J X, Jorda J L, Diaz-Cabanas M J, Yu J H, Corma A. Angew Chem, Int Ed, 2010, 49: 4986

59 Jiang J X, Jorda J L, Yu J H, Baumes L A, Mugnaioli E, Diaz-Cabanas M J, Kolb U, Corma A. Science, 2011, 333: 1131

60 Davis M E, Lobo R F. Chem Mater, 1992, 4: 756

61 Harrison W T A, Gier T E, Stucky G D, Broach R W, Bedard R A. Chem Mater, 1996, 8: 145

62 Feng P Y, Bu X H, Tolbert S H, Stucky G D. J Am Chem Soc, 1997, 119: 2497

63 Gier T, Bu X H, Feng P Y, Stucky G D. Nature, 1998, 395: 154

64 Tang L Q, Shi L, Bonneau C, Sun J L, Yue H J, Ojuva A, Lee B-L, Kritikos M, Bell R G, Bacsik Z, Mink J, Zou X D. Nature Mater, 2008, 7: 381

65 Roth W J, Dorset D L. Microporous Mesoporous Mater, 2011, 142: 32

66 Corma A, Corell C, Llopis F, Martínez A, Pérez-Pariente J. Appl Catal A, 1994, 115: 121

67 Guarino R F, Li C H. Hydrocarbon Eng, 1999, 4: 39

68 Maerz B, Chen S S, Venkat C R, Mazzone D N. Hydrocabon Tech Intern, 1996: 21

69 Rouleau L, Lacombe S, Alario F. Merlen E, Kolenda F, Magne-Drisch J. US 6337 063. 2002

70 Martens J A, Souverijns W, Verrelst W, Parton R, Groment G F, Jacobs P A. Angew Chem, Int Ed, 1995, 34: 2528

71 Souverijns W, Martens J A, Froment G F, Jacobs P A. $J$ Catal, 1998, 174: 177

72 Zones S I, Hwang S-J. Chem Mater, 2002, 14: 313

73 Zones S I. US 20080075 656. 2008

74 Bull I, Muller U. WO 2011064 186. 2011

75 Lee G S, Nakagawa Y, Zones S I. US 6156 290. 2000

76 Zones S I, Zhang G, Krishna K R, Biscardi J A, Marcantonio P, Vittoratos E. US 20050092651.2005
77 Blackwell C S, Broach R W, Gatter M G, Holmgren J S, Jan D-Y, Lewis G J, Mezza B J, Mezza T M, Miller M A, Moscoso J G, Patton R L, Rohde L M, Schoonover M W, Sinkler W, Wilson B A, Wilson S T. Angew Chem, Int Ed, 2003, 42: 1737

78 Knight L M, Lewis G J. Stud Surf Sci Catal, 2005, 154A: 171

79 Moscoso J G, Lewis G J, Gisselquist J L, Miller M A, Knight L M. US 6713041.2004

80 奈特 $\mathrm{L} \mathrm{M}$, 刘易斯 $\mathrm{G} \mathrm{J}$, 米勒 $\mathrm{M} \mathrm{A}$, 莫斯科索 $\mathrm{J} \mathrm{G}$, 吉塞 尔奎斯特 $\mathrm{J} \mathrm{L}$, 巴顿 R L, 威尔逊 $\mathrm{S} \mathrm{T}$, 詹登阳, 柯斯特 $\mathrm{S}$ C (Rohde L M, Lewis G J, Miller M A, Moscoso J G, Gisselquist J L, Patton R L, Wilson S T, Zhan Dengyang, Koster S C). CN 1997 593. 2007

81 Wang J Y, Song J W, Yin C Y, Ji Y Y, Zou Y C, Xiao F Sh. Microporous Mesoporous Mater, 2009, 117: 561

82 周朋燕, 李晓峰, 王裕金金, 武行洁, 窦涛. 石油学报 (石 油加工) (Zhou P Y, Li X F, Wang Y X, Wu X J, Dou T. Acta Petrol Sin (Petrol Process Sect)), 2008, 24(Z1): 226

83 McWilliams J P, Rubin M K. US 4894 212. 1990

84 Moini A, Valyocsik E W. US 5192521.1993

85 Zones S I, Holtermann D, Innes R A. US 5200377.1993

86 Nakagawa Y, Zones S I. US 5707 600. 1998

87 Nakagawa Y. US 5316 753. 1994

88 李赫咺, 项寿鹤, 吴德明, 刘月亭, 张晓森, 刘述铨. 高 等学校化学学报 (Li H X, Xiang Sh H, Wu D M, Liu Y T, Zhang X S, Liu Sh Q. Chem J Chin Univ), 1981, 2: 517

89 王福生, 程文才, 张式. 催化学报 (Wang F Sh, Cheng W C, Zhang Sh. Chin J Catal), 1981, 2: 282

90 Shiralkar V P, Clearfield A. Zeolites, 1989, 9: 363

91 孟祥举, 谢涁, 肖丰收. 催化学报 (Meng X J, Xie B, Xiao F Sh. Chin J Catal), 2009, 30: 965

92 Song J W, Dai L, Ji Y Y, Xiao F Sh. Chem Mater, 2006, 18: 2775

$93 \mathrm{Wu}$ Z F, Song J W, Ji Y Y, Ren L M, Xiao F Sh. Chem Mater, 2008, 20: 357

94 Xie B, Song J W, Ren L M, Ji Y Y, Li J X, Xiao F Sh. Chem Mater, 2008, 20: 4533

95 Kamimura Y, Chaikittisilp W, Itabashi K, Shimojima A, Okubo T. Chem Asian J, 2010, 5: 2182

96 Kamimura Y, Tanahashi S, Itabashi K, Sugawara A, Wakihara T, Shimojima A, Okubo T. J Phys Chem C, 2011, 115: 744

97 汪靖, 程晓维, 杨晓蔚, 龙英才. 化学学报 (Wang J, Cheng X W, Yang X W, Long Y C. Acta Chim Sin), 2008, 66: 769

98 Warrender S J, Wright P A, Zhou W Z, Lightfoot P, Camblor M A, Shin C H, Kim D J, Hong S B. Chem Mater, 2005, 17: 1272

99 Zhang H Y, Guo Q, Ren L M, Yang C G, Zhu L F, Meng X J, Li C, Xiao F Sh. J Mater Chem, 2011, 21: 9494

100 Yokoi T, Yoshioka M, Imai H, Tatsumi T. Angew Chem, Int Ed, 2009, 48: 9884

101 Xie B, Zhang H Y, Yang C G, Liu S Y, Ren L M, Zhang L, 
Meng X J, Yilmaz B, Muller U, Xiao F Sh. Chem Commun, 2011, 47: 3945

102 Kuznicki S M. US 4453 202. 1989

103 Kuznicki S M, Bell V A, Nair S, Hillhouse H W, Jacubinas R M, Braunbarth C M, Toby B H, Tsapatsis M. Nature, 2001, 412: 720

104 Taramasso M, Perego G, Notarir B. US 4410 501. 1983

105 Reddy J S, Kumar R, Ratnasamy P. Appl Catal, 1990, 58: L1

106 Camblor M A, Corma A, Martinez A, Perez-Pariente J. $J$ Chem Soc, Chem Commun, 1992: 589

107 van der Waal J C, Kooyman P J, Jansen J C, van Bekkum H. Microporous Mesoporous Mater, 1998, 25: 43

108 Tatsumi T, Jappar N. J Phys Chem, 1998, 102: 7126

109 Tuel A. Zeolites, 1995, 15: 236

110 Serrano D P, Li H X, Davis M E. J Chem Soc, Chem Commun, 1992: 745

111 Wu P, Komatsu T, Yashima T. J Phys Chem, 1996, 100: 10316

112 Dartt C B, Davis M E. Appl Catal A, 1996, 143: 53

113 Diaz-Cabanas M J, Villaescusa L A, Camblor M A. Chem Commun, 2000: 761

114 Wu P, Tatsumi T, Komatsu T, Yashima T. J Phys Chem B, 2001, 105: 2897

115 Wu P, Tatsumi T. Chem Commun, 2002: 1026

116 Wu P, Miyaji T, Liu Y M, He M Y, Tatsumi T. Catal Today, 2005, 99: 233

117 Kubota Y, Koyama Y, Yamada T, Inagaki S, Tatsumi $\mathrm{T}$. Chem Commun, 2008: 6224

118 Fan W B, Duan R G, Yokoi T, Wu P, Kubota Y, Tatsumi T. $J$ Am Chem Soc, 2008, 130: 10150

119 林民, 朱斌, 舒兴田, 汪故卿. 石油学报 (石油加工)(Lin M, Zhu B, Shu X T, Wang X Q. Acta Petrol Sin (Petrol Process Sect)), 2005, 34(Z1): 377

120 谢伟, 刘月明, 汪玲玲, 吴鹏. 催化学报 (Xie W, Liu Y M, Wang L L, Wu P. Chin J Catal), 2010, 31: 502

121 Bull I, Xue W M, Burk P, Boorse R S, Jaglowski W M, Koermer G S, Moini A, Patchett J A, Dettling J C, Caudle M T. US 7601662.2009

122 Andersen P J, Bailie J E, Casci J L, Chen H Y, Fedeyko J M, Foo R K S, Rajaram R R. WO 2008132 452. 2008

123 Houel V, James D, Millington P, Pollington S, Poulston S, Rajaram R, Torbati R. J Catal, 2005, 230: 150

124 Grossale A, Nova I, Tronconi E, Chatterjee D, Weibel M. Top Catal, 2009, 52: 1837

\section{英 译 文 \\ English Text}

In the chemical industry most processes (more than $80 \%$ ) involve catalysis especially in the petroleum and chemical industries. Catalysts are indispensable in many processes and zeolitic catalysts are important in these processes. The industrial application of zeolitic catalysts began in the 1960s when the FAU zeolite was the first to be used in the oil refining industry instead of the silica-alumina FCC catalyst by the former Mobil Company. Zeolitic catalysts are superior in terms of gasoline production and the utilization of crude oil compared to the conventional silica-alumina catalyst [1]. Currently, as a refinery catalyst, zeolitic catalysts occupy about $18.5 \%$ of the global catalyst manufacturing business [2]. Zeolitic catalysts have many distinctive advantages such as a regular pore system and a large inner micropore surface area, are comparable with $\mathrm{H}_{2} \mathrm{SO}_{4}$ and $\mathrm{AlCl}_{3}$, have molecular sieve or shape-selective properties, are amenable to modification or doping, etc. They have been widely used in many industrial catalysis processes including oil refinery and petroleum chemical processes such as catalytic cracking, hydrocracking, oligomerization, m-reforming, lube hydrotreating, disproportionation, isomerization, alkylation, aromatics transalkylation, olefins catalytic cracking, methanol to gasoline, aromatization of olefins, alkylation of benzene, propylene or ethylene, and some fine chemical or coal chemical processes such as selective catalytic oxidation and methanol to olefins conversion (Table 1). The highly efficient catalysis of zeolitic catalysts has been very profitable for the petroleum and chemical industry.

However, with the increase in petroleum product consumption and the shortage of crude oil resources worldwide in addition to changes in the composition and sources of raw materials (increase in the heavy compositions in the crude oil), coal, natural gas, and bio-ethanol have become alternative resources, etc. Additionally, with the increased demand for energy saving and environment protection, improvements and innovation in the petrochemical industry technology are highly desirable. The development of new technologies such as heavy crude oil deep processing, coal or other alternative resources chemical processing, highly efficient transformation of low value side products, synthesis of high value special petroleum products, energy saving and consumption reducing technology, $\mathrm{CO}_{2}$ chemical utilization, etc. is in demand. Most of these new technologies should involve innovations in the development of catalysts and in the development of zeolitic catalysts. From an industrial point of view, to achieve industrial goals such as high efficiency or atomic economic utilization of resources, energy savings and a reduction in consumption as well as environmental friendliness, many problems and challenges exist for the future development of zeolitic catalysts such as maximizing the efficiency of the zeolitic catalysts, the production of zeolitic catalysts by rational design, the economic synthesis of zeolites, and new applications of zeolitic catalysts. The main problems or challenges are listed in detail as follows.

(1) One of the challenges is the rational design of the zeolitic catalysts according to the desired catalytic reaction because different industrial catalytic reactions have different 
requirements for the catalysts and the requirements may often be influenced by a change in the resources and in the product market. The precise control of pore size and shape, morphology of crystallites, chemical composition of the zeolitic materials, acid strength and distribution, etc. are important.

(2) Another challenge is to improve the catalytic efficiency and the other properties of the zeolitic catalysts that are currently used in the industrial catalytic reactions. Many questions need to be answered such as the type of pores within the zeolites that improve performance and how to improve the utilization of pores and catalytic efficiency, whether zeolite pores can be changed or increased, the effect of framework heteroatoms on the catalytic performance, the development of surface chemistry or surface modifications on the zeolites, etc.

(3) The third challenge is to search for and synthesize new zeolites with improved catalytic performance, which may result in innovations in the developed processes and technologies. The discovery of new useful zeolite materials is relatively difficult and requires creative work to produce large pore zeolites with high hydrothermal stability, chiral zeolites and other molecular sieves with novel structures, etc. Although there are almost 190 kinds of zeolite topologies the individual features, the kinds of zeolites that have higher catalytic performance and the kinds of zeolites that have potential application in industry need to be studied in detail.

(4) Some zeolites show excellent performance and these are potentially useful in industry. However, their production costs are currently too high because they are synthesized using expensive organic structural directing agents (SDA). Therefore, the economic synthesis of these zeolites or a reduction in their synthesis costs is a big challenge.

(5) Studying new applications of zeolites in fields such as the fine chemical industry, green chemistry or environmental protection, etc. is also of interest.

We thus review some recent progress as well as discuss the development of zeolitic catalysts according to the challenges and questions listed above.

\section{Highly efficient catalysis of zeolitic catalysts}

For industrial zeolites, high performance is a basic requirement. The type and amount of active centers as well as the diffusive properties of micropores are intrinsic factors for catalytic materials that determine their catalytic activities. Catalytic selectivity is closely related to the shape-selectivity of micropores, the side reactions, and the molecular diffusion rates. Lifetime is an important property of a catalyst and prolonging their lifetime is always important. If the catalytic activity of a catalyst is high enough and the catalyst cokes easily it can still be used for long periods in a suitable reac- tion process if it has good generation properties. Therefore, good performance is not only related to the catalytic materials but also to a combination of the reaction process and the engineering system, which helps in obtaining good performance for the zeolitic catalysts in real industrial applications.

The following is a discussion about improving the catalytic efficiency of the zeolitic catalysts.

\subsection{Crystal size and morphology control}

Because the micropore size of most of zeolites is often less than $1 \mathrm{~nm}$, the diffusion of small organic molecules would be limited by their pore channels, which may result in inadequate pore utilization and a relatively low catalytic activity. Decreasing the zeolite crystal size or changing their morphology should be effective in improving the diffusion properties and utilization of pores. Small or nano crystallites should have a shorter diffusion path and a larger extent of pore utilization than large crystallites, which would result in better catalytic performance. Therefore, since the 1990s studies on nanozeolites have aroused great interest in this field and many nanozeolites with LTA [5], LTL [6], MFI [7], BEA [8] structures, etc. have been successfully synthesized. Nanozeolites show better catalytic performance than regular-size zeolites [9].

SAPO-34 molecular sieves with a CHA topology are considered to be one of the most effective industrial catalysts for the methanol to olefins (MTO) conversion process. Recently, Yang et al. [10] studied the effect of crystal size and morphology of SAPO-34 on the production of olefins and the lifetime of catalysts. They found that the crystal size and morphology of SAPO-34 greatly influenced their catalytic performance, for example, smaller or lamellar crystallites gave better catalytic performance (higher yield of $\mathrm{C}_{2}$ and $\mathrm{C}_{3}$ olefins and working over longer periods) than larger crystallites.

Recently, Choi et al. [11] successfully synthesized multior single-lamellar MFI nanosheets that were created by the coherent assembly of a zeolite layer and a structure directing agent as well as a diquaternary ammonium surfactant with relatively long hydrocarbon chains. These zeolite nanosheets were found to have a much lower deactivation or coking rate for the conversion of methanol to gasoline (MTG).

However, many problems are evident during the synthesis and use of small particle- or nanozeolites, for example, they agglomerate easily during their synthesis process, which requires the carefully control of gel composition and synthesis conditions. They also agglomerate easily during drying and calcination, which may reduce their catalytic performance. The synthesis of nanozeolites often involves the use of expensive organic SDA, which may result in a low 
production yield and high cost. In addition, when using nanozeolites, especially in a liquid catalytic system, the nanoparticles are not easy to separate from the liquid products, which may result in recycling difficulties. All these difficult problems mentioned need to be resolved before their practical application.

\subsection{Zeolites with hierarchical pores}

Zeolite-based hierarchical porous materials with two or more levels of porosity, especially mesoporous zeolites have received much attention recently. Most reported mesoporous silicas or aluminosilicates have properties such as unsatisfied thermal stability, are just short of being strong acids and easily lose acid centers because their walls are not crystalline but are amorphous although their pores may be periodic and regular. While zeolites have good structural stability and are strong acids they have a molecular diffusion limitation, which influences their catalytic activity and selectivity. However, hierarchical porous zeolites may combine the advantages of common zeolites with efficient mass transport and large porous molecular sieves. Recently, some hierarchical porous zeolites have been synthesized using carbon black [12], carbon nanotubes [13], starch [14], nanosized $\mathrm{CaCO}_{3}$ [15], polymers (PDDA[16], PVB [17], etc.), and amphiphilic organosilane surfactants [18] as templates. Upon the direct hydrothermal crystallization of templates/silica composites the silica was transformed into zeolite whereas the templates were incorporated in the zeolite crystals. The removal of the templates inside the zeolite composite resulted in the opening of micropores and mesopores in the zeolites, respectively. An alternative route for hierarchical porous zeolites with crystalline walls is the recrystallization of the walls of SBA and MCM materials in the presence of molecular templates [19].

Hierarchical porous zeolites give better catalytic performance in reactions such as supercritical toluene disproportionation [20], alkylation of benzene with ethylene [12], and the transalkylation of heavy aromatics [21]. Recenty, Yang et al. [22] prepared a hierarchical beta zeolite by the dry gel conversion method. They found that the hierarchical beta zeolite gave better activity and had better stability than the conventional beta zeolite for the transalkylation reaction of diisopropylbenzene with benzene.

Therefore, it is expected that hierarchical porous zeolites may have application in large molecule catalysis or in liquid catalytic reaction systems in the future.

\subsection{Composite zeolites by co-crystallization or intergrowth}

During the synthesis of zeolites, a pure zeolite phase can be obtained only when the synthesis parameters are well controlled while a variation in zeolite synthesis conditions will lead to the formation of co-crystallized zeolites with different phases. This is called co-crystallization. These mixtures may be regarded as physical mixtures in many cases and their structures and properties have been neglected. Only a few examples of the intergrowth zeolites from co-crystallization such as the intergrowth of zeolites X/A, MFI/MEL, FAU/EMT, and STF/SFF with their analogous basic structures have been reported. Some co-crystallized zeolites show better performance than the individual pure phases, for example, ZSM-5/ZSM-11 (MFI/MEL) is a kind of intergrowth zeolite and it can be used to adjust the product composition of gasoline over a wide range in the methanol to gasoline conversion process [23]. The composite zeolites obtained by co-crystallization or intergrowth can have precisely adjusted pore and acid systems, which may be effective in improving their catalytic performance.

Recently, Xie et al. [24] carried out a series of investigations on MCM-49/ZSM-35 that was synthesized by the crystal transformation of MCM-49 or in a mixed amine system containing hexamethyleneimine and cyclohexamine. They found that the MCM-49 and ZSM-35 analogues in the co-crystallized zeolites may be stacked much closer than those in the physical mixture and some parts of the intergrowth may be formed because of the partially similar basic structure of MCM-49 and ZSM-35. They also observed a notable synergistic effect during the FCC gasoline updating process in which MCM-49/ZSM-35 showed a higher aromatization ability than pure MCM-49 [25]. They also observed a similar synergistic effect for MCM-49/ZSM-35 during aromatization in 1-butene conversion. Even though a small amount of MCM-49 was present in MCM-49/ZSM-35 this co-crystallized zeolite showed a relatively high aromatization conversion [26].

Qi et al. [27] investigated BEA/MOR (a co-crystalline zeolite of beta and mordenite) zeolite and they found enhanced acid properties and catalytic activity for methanol dehydration compared with the individual pure beta or mordenite zeolite.

Co-crystallized or intergrowth zeolites have not been fully studied and their special intergrowth structures, properties, and catalytic performances need to be investigated further.

\subsection{Surface modification of zeolite for better hydrothermal stability}

Thermal stability and hydrothermal stability are important properties of a zeolitic catalyst. Many industrial reactions require high thermal stability and high hydrothermal stability, which is often important for the life of the catalyst and it determines the selection of the catalytic process. 
For example, the catalytic cracking of mixed $\mathrm{C}_{4^{-}}$or $\mathrm{C}_{5}$-olefins to produce propylene and ethylene requires a steam atmosphere and, therefore, the hydrothermal stability of the zeolite catalysts is very important for catalyst development. It has been reported that phosphorus modification or the incorporation of heteroatoms are effective methods to increase the hydrothermal stability of zeolites [28]. Zhao et al. [29] prepared a series of catalysts for $\mathrm{C}_{4}$-olefin cracking by impregnating HZSM-5 with different amounts of phosphoric acid followed by calcination in air at high temperature. The introduction of a suitable amount of phosphorus into the HZSM-5 zeolite can slow down coke deposition and dealumination, which are the main causes of HZSM-5 deactivation. A synergistic interaction may exist between the phosphorus oxide species and the Si-O-Al framework within the zeolite pore channels, which is good for optimizing the acid strength and distribution, reducing the side reactions of hydrogen transfer, and increasing the selectivity for propylene. To describe the exact structure of the new acids that form in P/HZSM-5 during steam treatment Xue et al. [30] used $\mathrm{D}_{2} / \mathrm{OH}$ exchange and solid-state ${ }^{31} \mathrm{P}$ and ${ }^{27} \mathrm{Al} \mathrm{NMR}$ measurements to investigate the change in acid centers before and after phosphorus-modification. They also proposed a model with the assistance of a computer simulation. They found that the new acid sites that were generated by phosphorus-modification seem to be related to phosphorus entering the zeolitic at the position left by dealumination and stabilized by some extra-lattice aluminum. The introduction of phosphorus to the zeolitic framework protects the residual framework aluminum against further dealumination. The integrated effect results in hydrothermally stable acid sites in P-modified HZSM-5 and enhances the catalytic performance toward $\mathrm{C}_{4}$ olefin cracking after steam treatment at high temperature.

For the methanol dehydration to olefins, a large amount of water is generated in the product mixtures and, therefore, the zeolitic catalyst should be able to withstand steam treatment and regeneration at high temperatures. Because the SAPO-34 zeolite has good thermal and hydrothermal stability it has been the catalytic material of choice for the MTO process.

For naphtha cracking to olefins the reaction temperature often reaches $600{ }^{\circ} \mathrm{C}$ and, therefore, the thermal and hydrothermal stability of the catalyst are very important.

Many other refining and petrochemical reactions require catalysts with good thermal and hydrothermal stability. The search for highly stable catalytic materials and methods to improve their stability are essential subjects and are worth studying.

\section{Synthesis and application of zeolites with novel structures}

\subsection{Large or extra-large pore molecular sieves}

Although zeolitic catalysts have been widely used in many small molecular reactions in the petrochemical and fine chemical industry, they are hardly used in large molecule (e.g. heavy compositions of crude oil, fine chemicals, or organic intermediates) catalysis because of their small micropore size. Therefore, the synthesis of large (12-membered rings (MR)) or extra-large pore (12-MR above) zeolites has been an important subject over the last 30 years (Table 2).

VPI-5, an aluminophosphate molecular sieve, is the first example of a large pore molecular sieve and its pore size exceeds $1 \mathrm{~nm}$. It has a large pore size of $1.2 \mathrm{~nm}$ and a $30 \%$ void ratio. This discovery of large pore zeolites opened a new area in the zeolite research field. Many microporous materials with large or extra-large pores (including aluminosilicates, aluminophosphates, germanosilicates, etc.) have been synthesized or discovered (Table 2). Among these large or extra-large pore molecular sieves, aluminophosphates have a relatively low framework stability, e.g. JDF-20 and ND-1 collapse after the removal of the organic templates within the pores. Large or extra-large pore aluminosilicates (or zeolites) have fairly good stability, e.g. UTD-1 and CIT-5 have comparable stability to that of small pore zeolites [42]. Therefore, from a stability point of view, large or extra-large pore zeolites are more useful than aluminophosphates in future industrial applications.

Corma's group has synthesized a series of germanosilicates including some large or extra-large pore silicates such as ITQ-21 [50], ITQ-24 [51], ITQ-33 [55], ITQ-43 [59], ITQ-44 [58], etc. Among them, ITQ-21 has a three-dimensional channel system with circular openings comprising 12-membered rings and three linear channels that intersect to produce large inner cavities, and every cavity can be entered into through six windows. ITQ-21 was found to be more active than USY for catalytic cracking and produced more propylene in the gas fraction while giving less olefinic gasoline with a higher RON and MON [50]. ITQ-33 possesses straight large pore channels with circular openings of 18-rings along the $c$ axis interconnected in a bidirectional system that consists of 10-ring channels. This material is very active during the alkylation and transalkylation of alkyl aromatics as well as during the dealkylation of bulky alkylaromatics containing one or two condensed aromatic rings. It is also good for producing more diesel and less gasoline while maintaining the propylene and butene yield during the catalytic cracking of vacuum gasoil [55].

Therefore, if the stability and economy of synthesis were further improved, it is expected that large or extra-large pore zeolites such as ITQ-21 and ITQ-33 may be applied to the refining and petrochemical industries in future. 


\subsection{Chiral molecular sieves}

Highly selective reactions including asymmetric catalytic reactions have become important research topics and may have potential applications in pharmaceuticals, agriculture chemicals, food additives, etc. A zeolitic asymmetric catalyst may be prepared by the immobilization of chiral coordination compounds or metallo-organic compound catalysts on zeolite supports. However, the stability and lifetime of these catalysts is often unsatisfactory for real applications because of the relatively weak bonds of the chiral group with the surface of the zeolite. When chiral zeolites are synthesized the stability of the catalysts are readily determined. Davis et al. [60] synthesized chiral beta zeolite templated by a chiral organic amine but the enantiomer selectivity of the beta zeolite was only $5 \%$, which was identified by a light reaction test. Stucky's group synthesized $\mathrm{NaZnPO}_{4}$ [61], $\mathrm{NaCoPO}_{4}$ [62], and gallium germanate UCSB-7 [63] with chiral structures or pores. Recently, Zou's group [64] reported two chiral germanosilicates SU-15 and SU-32. Although practical application of these chiral molecular sieves has not been achieved, good basic research into their chiral catalysis has been carried out.

\subsection{Other zeolites with novel structures}

MWW zeolite is a new kind of silica layered zeolite that contains large zeolites including MCM-22, MCM-49, MCM-36, MCM-56, PSH-3, SSZ-25, ERB-1, ITQ-1, ITQ-2, EMM-10, EMM-12, EMM-13 [65], etc. Its framework topology consists of two independent pore systems, one formed by two-dimensional sinusoidal channels and the other by large supercages $(0.71 \mathrm{~nm} \times 0.71 \mathrm{~nm} \times 1.82 \mathrm{~nm})$. MWW zeolite is different to other zeolites as it has a high concentration of external pockets with 12-ring openings and an approximate depth of $0.71 \mathrm{~nm}$. Because of its unusual crystalline structure, high microporosity, highly hydrothermal stability, and mild acid performance it is not surprising that the MWW zeolite has good catalytic performance properties for many reactions such as olefin isomerization, alkylation for ethylbenzene or cumene production, catalytic cracking or hydrocracking, disproportionation of toluene, aromatization, etc. [66]. Therefore, studies into the MWW zeolite have been a popular zeolite research area over the last 20 years. One big commercial application of the MCM-22 zeolite catalyst has been introduced by the Exxon-Mobil Company for the production of cumene by the liquid-phase alkylation in the new Mobil/Badger-Raytheon process [67]. This process has a low reaction temperature and the catalyst has a long lifetime while high selectivity for cumene products is obtained $(99.99 \%)$. Therefore, this process was quickly substituted for old processes based on zeolite ZSM-5 or beta zeolite worldwide. In addition, MWW zeolite has also been used industrially in ethylbenzene production [68].

EU-1 (EUO) zeolite with 10-MR straight pore channels and vertically connected side pockets has better catalytic performance, activity, and selectivity toward xylene isomerization [69]. Therefore, the EU-1 zeolite is a good candidate for a new generation of industrial catalysts for xylene isomerization.

Medium pore zeolites with unconnected one dimensional pore channels such as TON, MTT, EUO, AEL, etc. have better production or selectivity as dual functional hydrocarbon hydroisomerisation catalysts than conventional ZSM-5, ZSM-11, or beta zeolite and may be applied industrially in the future $[70,71]$.

\section{Economic synthesis of zeolites}

Some zeolites may be synthesized in an inorganic system with inorganic cations e.g. zeolite A, P, Mordernite, X, Y, and L. Other zeolites need to be synthesized using organic SDAs such as ZSM-5, ZSM-11, beta, MCM-22, SAPO-34, and TS-1. Compared with the zeolite synthesized in inorganic systems, zeolite products that are prepared by organic SDA often have better crystallinity, crystal size, and $\mathrm{Si} / \mathrm{Al}$ ratios, and these properties are also easier to control. However, their cost is much higher. Many new zeolites are often synthesized using expensive organic SDAs and their production costs are very high. Therefore, much effort has been put into reducing the production cost of zeolites by the substitution of expensive organic SDAs with cheaper and environmental friendly SDAs or by organic template-free synthesis.

\subsection{Substitution of the organic structure directing agents}

It has been recognized that the organic SDAs used is in excess of that occluded in zeolite crystals, which means that only a minor portion of the SDA that was used specifies the nucleation and growth of the zeolite product while in the rest a large portion of amine was used to provide both pore filling and basicity capacity in the synthesis. Zones et al. [72] developed a new approach by adding multi-organic amines and one of these amines acted as a SDA while other cheaper amines were used to fill pores and to adjust the basicity. In one example, except for the addition of quaternized aminoadamantane, which was needed for the synthesis of SSZ-25 (MWW) a cheap isobutylamine was also used to partly substitute for expensive aminoadamantane. This synthesis route offers cost-saving benefits by reducing the costs associated with SDAs, waste stream cleanup costs as well as the time in the reactor and the reagent flexibility was increased too. 
Similarly, they synthesized SSZ-13 (CHA), SSZ-33 (CON), SSZ-35 (STF), and SSZ-42 (IFR) [73] by partly substituting expensive aminoadamantane with $\mathrm{N}, \mathrm{N}, \mathrm{N}$ trimethyl aniline. Bull et al. [74] synthesized SSZ-13 by combining aminoadamantane and tetramethylammonium hydroxide. The multi SDAs method not only reduces the cost but also has the added benefit of adjusting the phase composition, the $\mathrm{Si} / \mathrm{Al}$ ratio, and the crystal size of the product. One example is SSZ-47, which is a complex intergrowth of the three framework types; EUO, NES, and NON. The presence of NON in this intergrowth can greatly reduce the sorption capacity and the ease of diffusion. SSZ-47B is prepared using a combination of two SDAs and it has a relatively lower level of NON, which results in a much higher sorption capacity [75]. Another example is SSZ-32X (MTT), which has also been prepared using the two SDAs method [76]. Compared to the SSZ-32 prepared using imidazolium SDA the SSZ-32X has a reduced $\mathrm{Si} / \mathrm{Al}$ ratio and a reduced crystal size as well as better dewaxing catalytic performance.

MCM-22 (MWW) is a new kind of industrial zeolite and it is now mainly produced using toxic hexamethyleneimine as the SDA. Lewis et al. [77-80] prepared a series of UZM zeolites using a combination of commercially available SDAs. Among them, UZM-8 (MWW) was prepared using tetraethylammonium hydroxide $(\mathrm{TEAOH})$ and $\mathrm{Et}_{2} \mathrm{Me}_{2} \mathrm{NOH}$ as the SDAs [80], which is advantageous in terms of their production cost and environmentally friendliness when compared to hexamethyleneimine as the SDA.

Wang et al. [81] reported the successful synthesis of AFI aluminophosphate and its heteroatom compounds using cheap and environmentally friendly tetramethylguanidine as the SDA.

\subsection{Organic template-free synthesis}

A low cost organic structure directing agent (OSDA) or alternatively organic-free synthesis is desired in industry.

The addition of crystalline seed to the preparation system has been shown to be an effective method to decrease the OSDA. EU-1 [82], ZSM-11 [83], ZSM-12 [84], SSZ-28 [85], SSZ-32 [86], and SSZ-35 [87] were synthesized by this method with a $\mathrm{SDA} / \mathrm{SiO}_{2}$ molar ratio as low as $0.02-0.05$. However, obtaining a synthesis route without OSDA is still a challenge.

In the 1980s a new route to prepare ZSM-5 without OSDA was developed and subsequently applied in industry by $\mathrm{Li}$ and Xiang et al. [88]. Wang et al. [89] and Clearfield et al. [90] then synthesized the ZSM-5 zeolite under an ammonium environment using amorphous aluminum silicate as a precursor. Recently, Xiao et al. [91] developed an amine-free strategy to prepare zeolites in which zeolite seeds or a gel initiating agent was added to promote the growth of zeolite crystals. By adding a gel initiating agent such as zeolite Y, zeolite L, or calcined beta zeolite seed ECR-1 [92], ZSM-34 [93], and beta zeolites [94] were synthesized without OSDA. However, the $\mathrm{Si} / \mathrm{Al}$ ratio of the beta zeolite product was relatively low. Furthermore, Kaminura et al. [95] tried to increase the $\mathrm{Si} / \mathrm{Al}$ ratio of the beta zeolite and investigated the crystal formation process of OSDA in detail. They suggested that the beta seeds in the OSDA-free $\mathrm{Na}^{+}$-aluminosilicate gel system provided a growth surface for new beta crystals but they did not induce the nucleation of new beta crystals [96].

Using the above-mentioned OSDA-free method, many low silica zeolites including FAU, MOR [97], AMA, CHA, and high silica zeolites including ZSM-5, TUN-7 [98], ECR-1 [92], ZSM-34 [93], Ferrierite [99], beta [94,95], RTH [100], MTW [101], etc. have been synthesized.

Economic and environmental advantages give the OSDA-free route more of a chance to be taken up in industry. However, compared with the conventional OSDA strategy some challenges still exist. First, during OSDA-free synthesis it takes a relatively long period to achieve highly crystalline zeolite products despite seed being used. Second, the range of the $\mathrm{Si} / \mathrm{Al}$ ratio is relatively narrow and it is not easily adjusted by the OSDA-free method. Third, without the effect of OSDA the prevention of impure crystal growth and the modulation of crystal size are challenging. Although it has been reported that some trials were conducted on the OSDA-free method in industry [100] more technologies should be developed to solve the above problems.

\section{New applications}

With the increase in zeolite research more applications for zeolites have been developed in various fields such as adsorption, ion-exchange, gas separation, selective catalytic oxidation, selective catalytic reduction of $\mathrm{NO}_{x}$, and other green processes.

ETS-4, which was found by Kuznicki et al. [102] in 1989 is a kind of microporous titanosilicate that possesses tetrahedral silicon atoms and octahedral titanium atoms in its framework. The ETS-4 framework can be systematically contracted by dehydration at elevated temperature to "tune" the effective size of the pores and this is referred to as the molecular gate effect [103]. Using this special characteristic, ETS-4 has been used by the Engelhard Corporation to separate $\mathrm{N}_{2}$ and $\mathrm{CO}_{2}$ from natural gas during its transportation by pipeline.

Moreover, microporous titanosilicates such as TS-1 [104], TS-2 [105], Ti-MWW etc. have been used as catalysts for selective oxidation reactions such as the oxidation of phenol to hydroquinone, the oxidation of cyclohexanone ammonia 
to cyclohexanone oxime, the epoxidation of propylene etc., using $\mathrm{H}_{2} \mathrm{O}_{2}$ as an oxide, and these are new green processes. Over the past few decades, many researchers have paid attention to the synthesis of new titanosilicates such as Ti-beta [106-108], Ti-ZSM-12 [109], Ti-ZSM-48 [110], Ti-mordenite [111], Ti-SSZ-33 [112], Ti-ITQ-7 [113], Ti-MCM-22 (Ti-MWW) [114-116], Ti-MCM-56 [117] etc., to try increase the amount of tetrahedral titanium atoms in the framework [118] and, furthermore, to explore industrial applications. In China, Research Institute of Petroleum Processing (RIPP) of SINOPEC and East China Normal University have done much work on titanosilicates e.g. the production of hollow TS-1 [119] and Ti-MWW [120], which has encouraged us to look forward to more applications of titanosilicate zeolites in the green chemistry industry.

Zeolite is also available as an effective catalyst in the environmental field. The highly efficient selective reduction of $\mathrm{NO}_{x}$ from the emission of lean burn engines and diesel engines has attracted much attention all over the world recently. It has been reported that zeolites that contain transition metal ions such as $\mathrm{Cu} / \mathrm{SAPO}-34$ [121], Cu/Beta [122], Fe/Beta [122], Cu/ZSM-5 [123] et al., demonstrate high efficiency and selectivity in the selective catalytic reduction of $\mathrm{NO}_{x}$ [124]. More applications for zeolitic catalysts are expected.

\section{Prospects for zeolites and zeolitic catalysis}

The application of zeolites in industry as catalysts began 50 years ago. Over the past few decades zeolites have played important roles in oil refining, petrochemicals, and in separations and they have made chemical processes more effective and economic. However, over the last decade more challenges have arisen in the development of the conventional petrochemical industry. Awareness about energy con- servation and environmental protection by the reduction or transformation of greenhouse gases is important. Therefore, much attention has been paid to the development of new chemical conversion routes from coal, natural gas, and biomass resources. Increasing the efficiency of zeolites as catalysts, designing and synthesizing catalysts depending on demand and more importantly the development of economic routes and more applications of zeolites has been a focus.

With the further development of zeolite chemistry more zeolites with new structures will be synthesized and this will enrich the types of zeolites and also lead to a greater amount of choice in terms of catalysis routes or processes in industry. Old industrial zeolites will be replaced by new zeolites with high performance, which will help increase the efficiency and economy of the old processes. New catalytic processes may also be developed because of the new zeolitic catalysts. In addition, as for conventional zeolites new manufacturing technologies to control the crystal size, the pore size and morphology such as in nanosized zeolites, the hierarchical porous zeolites, and special morphology zeolites such as lamellar and needle-like will advance improvements in catalysis processes. On the other hand, it is expected that chiral zeolites will be developed in fields such as fine chemicals, medicines, pesticides, and separations in future and this may be of interest to the chemical industry. Besides the petrochemical industry, zeolites will find more application in green chemistry and in environmental applications such as in the selective oxidation, selective catalytic reduction of $\mathrm{NO}_{x}$, etc. With the development of technologies and the reduction in costs, zeolites will play more important roles in industry and in our lives.

Full-text paper available online at Elsevier ScienceDirect http://www.sciencedirect.com/science/journal/18722067 